\title{
Abnormal Sensory Perception or Peristaltic Dysfunction: Which One Is Associated With Symptoms?
}

(Am J Gastroenterol 2014;109:521-526)

\section{Jung Ho Park}

Department of Internal Medicine, Kangbuk Samsung Hospital, Sungkyunkwan University College of Medicine, Seoul, Korea

\section{Summary}

The generation of esophageal symptoms while swallowing is a multifactorial phenomenon. Previous studies tried to analyze the correlation between objective esophageal functions using high-resolution manometry (HRM) and abnormal esophageal perceptions such as pain or dysphagia, but they were unable to verify the relationship between them.

Recently, Xiao et al ${ }^{1}$ investigated whether new manometric metrics can show a relationship between symptoms and abnormal motor function while swallowing. HRM studies included $10 \mathrm{su}-$ pine liquids, 5 upright liquids, 2 upright viscous and 2 upright solid swallows. All patients evaluated their esophageal symptoms for each upright swallow. Symptoms were graded on a 4-point likert score ( 0 , none; 1 , mild; 2 , moderate; 3 , severe). HRM metrics were compared between groups with and without symptoms during the upright liquid protocol. Of the 269 patients, 116 recorded symptoms during viscous or solid swallows. HRM metrics were similar between swallows with and without associated symptoms in the upright, viscous and solid swallows. No correla- tion was noted between the HRM metrics and the symptom scores among swallow types. Finally, they concluded that esophageal symptoms are not related to abnormal motor functions defined by HRM. Also, other factors beyond circular muscle contraction patterns should be explored as possible causes of symptom generation.

\section{Comments}

Until now, it was a common belief that abnormalities in contractility and deglutitive inhibition may correlate with dysphagia; however, previous studies were unable to verify the relationship between esophageal contractile abnormalities, bolus transport and dysphagia. In the study evaluating the relationship between transits of swallowed boluses using videofluoroscopy and the symptoms of dysphagia, self-reported dysphagia was rarely connected with stasis and patients frequently report dysphagia when bolus clearance was successful. ${ }^{2}$ Also, there was no congruency between measurements with manometry-impedance and subjective perception of the bolus passage. ${ }^{3,4}$ Instead, esophageal motor abnormalities and impaired bolus transits increase in harmony

Received: May 26, 2014 Revised: June 11, 2014 Accepted: June 13, 2014

(c) This is an Open Access article distributed under the terms of the Creative Commons Attribution Non-Commercial License (http://creativecommons. org/licenses/by-nc/3.0) which permits unrestricted non-commercial use, distribution, and reproduction in any medium, provided the original work is properly cited.

*Correspondence: Jung Ho Park, MD Department of Internal Medicine, Kangbuk Samsung Hospital, 29, Saemunan-ro, Jongno-Ku, Seoul 110-746, Korea

Financial support: None. Tel: +82-2-2001-2059, Fax: +82-2-2001-2485, E-mail: pjho3@hotmail.com

Conflicts of interest: None.

ORCID: http://orcid.org/0000-0003-2568-128X. 
with the severity of gastroesophageal reflux disease. ${ }^{5,6}$

Xiao et $\mathrm{al}^{1}$ suggested possible explanations for this discordance. Firstly, manometrical esophageal dysmotility is only an epiphenomenon with visceral hyperalgesia and sensori-motor dysfunction of the esophagus playing a key role in the symptom generation. ${ }^{7}$ Although the occurrence of chest pain could be explained in this manner, it is not clear that the symptom "dysphagia" arise in the same way as chest pain do. Secondly, there was the possibility of inaccurate measuring methods of relevant motor disorders. Instead of the circular muscle, the longitudinal muscle contraction can play an important role in the generation of heartburn and chest pain. ${ }^{8}$ Also, the new methodology utilizing combined impedance and manometry may be helpful clarifying the role of elevated intrabolus pressure in dysphagia. However, the significance of the role played by longitudinal muscle contraction in the development of chest pain was verified only in the patients with achalasia and also, I am doubtful whether longitudinal muscles play an important role in the occurrence of the symptom, dysphagia.

Personally, I think that the influence of the manometry catheter on the symptom reporting may be the most important confounding factor among the qualifications of this study. The catheter itself can cause very severe discomforts such as foreign body sensation, coughing and difficulty in eating. Therefore, it might be better to perform sensation studies separately from the manometric evaluation on the same day. Moreover, as a provocative test, responses to multiple rapid swallow are highly reproducible ${ }^{9}$ and reflect the esophageal body peristaltic reserve. ${ }^{10}$ Therefore, along with viscous swallowing, additional protocol with multiple rapid swallow could produce more interesting results.

From now on, when patients complain of "dysphagia" in outpatient clinics, we should consider 2 possible causes for their symptoms: the hindrance of food material and sensations having particles stuck in the esophagus. In the future, new techniques should be explored that may improve ability to assess esophageal hypersensitivity and bolus transit, just like the conclusion of Xiao et al. ${ }^{1}$

\section{References}

1. Xiao Y, Kahrilas PJ, Nicodeme F, Lin Z, Roman S, Pandolfino JE. Lack of correlation between HRM metrics and symptoms during the manometric protocol. Am J Gastroenterol 2014;109:521-526.

2. Bogte A, Bredenoord AJ, Oors J, Siersema PD, Smout AJPM. Sensation of stasis is poorly correlated with impaired esophageal bolus transport. Neurogastroenterol Motil 2014;26:538-545.

3. Lazarescu A, Karamanolis G, Aprile L, De Oliveira RB, Dantas R, Sifrim D. Perception of dysphagia: lack of correlation with objective measurements of esophageal function. Neurogastroenterol Motil 2010;22:1292-1297, e1336-e1337.

4. Chen CL, Yi CH. Clinical correlates of dysphagia to oesophageal dysmotility: studies using combined manometry and impedance. Neurogastroenterol Motil 2008;20:611-617.

5. Savarino E, Gemignani L, Pohl D, et al. Oesophageal motility and bolus transit abnormalities increase in parallel with the severity of gastro-oesophageal reflux disease. Aliment Pharmacol Ther 2011; 34:476-486.

6. Cho YK, Choi M-G, Lim CH, et al. Impaired esophageal bolus transit in patients with gastroesophageal reflux disease and abnormal esophageal acid exposure. Gut Liver 2012;6:440-445.

7. Nasr I, Attaluri A, Hashmi S, Gregersen H, Rao SSC. Investigation of esophageal sensation and biomechanical properties in functional chest pain. Neurogastroenterol Motil 2010;22:520-526, e116.

8. Hong SJ, Bhargava V, Jiang Y, Denboer D, Mittal RK. A unique esophageal motor pattern that involves longitudinal muscles is responsible for emptying in achalasia esophagus. Gastroenterology 2010; 139:102-111.

9. Price LH, Li Y, Patel A, Prakash Gyawali C. Reproducibility patterns of multiple rapid swallows during high resolution esophageal manometry provide insights into esophageal pathophysiology. Neurogastroenterol Motil 2014;26:646-653.

10. Shaker A, Stoikes N, Drapekin J, Kushnir V, Brunt LM, Gyawali CP. Multiple rapid swallow responses during esophageal high-resolution manometry reflect esophageal body peristaltic reserve. Am J Gastroenterol 2013;108:1706-1712. 